山्यRANÇAISE

$>\mathrm{DE}$

$\stackrel{1=1}{\simeq}$ PÉDAGOGIE
Revue française de pédagogie

Recherches en éducation

$195 \mid 2016$

Varia

\title{
Aux origines du Conseil national des programmes (1983-1990) : instruments et acteurs d'une nouvelle gouvernance des contenus d'enseignements
}

The origins of the National Council of Programs (1983-1990): instruments and actors of a new governance of the contents of lessons

Lydie Heurdier et Pierre Clément

(2) OpenEdition

Journals

Édition électronique

URL : http://journals.openedition.org/rfp/5016

DOI : 10.4000/rfp.5016

ISSN : 2105-2913

Éditeur

ENS Éditions

Édition imprimée

Date de publication : 30 juin 2016

Pagination : 5-24

ISSN : 0556-7807

Référence électronique

Lydie Heurdier et Pierre Clément, « Aux origines du Conseil national des programmes (1983-1990): instruments et acteurs d'une nouvelle gouvernance des contenus d'enseignements ", Revue française de pédagogie [En ligne], 195 | 2016, mis en ligne le 30 juin 2019, consulté le 08 janvier 2021. URL : http://journals.openedition.org/rfp/5016 ; DOI : https://doi.org/10.4000/rfp.5016 


\title{
Aux origines du Conseil national des programmes (1983-1990) : instruments et acteurs d'une nouvelle gouvernance des contenus d'enseignements
}

Lydie Heurdier Pierre Clément

\begin{abstract}
La création du Conseil national des programmes (CNP) en 1990 marque une double rupture dans l'histoire des réformes des programmes scolaires. La première tient à la temporalité et au cadre institutionnel des transformations des programmes : le CNP est l'instrument d'une réforme coordonnée, globale et simultanée de l'ensemble des disciplines en fonction de principes communs et applicables de manière transversale. La seconde tient à une modification des rapports de force entre les acteurs des réformes : la création du CNP se traduit par le dessaisissement de l'Inspection générale et des associations de spécialistes au profit du CNP, du cabinet du ministre et des directions pédagogiques du ministère. Pour expliquer cette double rupture, cet article étudie les dispositifs de légitimation, commissions et rapports, ainsi que les acteurs, individuels et collectifs, ayant présidé à la création du CNP. II montre ce que l'efficacité symbolique de ces dispositifs doit à leur approche systémique et globale des contenus, au soutien politique fort dont ils bénéficient, à leur ouverture à des acteurs d'ordinaire exclus du débat sur les programmes et à la mobilisation de la légitimité scientifique venant concurrencer la légitimité pédagogique et disciplinaire. II met enfin au jour les contradictions d'un dispositif oscillant entre autonomie et instrumentalisation politique de la légitimité scientifique et leurs conséquences sur le futur CNP.
\end{abstract}

Mots-clés (TESE) : réforme des programmes d'études, contenu de l'éducation, politique et administration de l'enseignement, expert en éducation, France 
Entre la fin de la Seconde Guerre mondiale et la deuxième moitié des années 1970, la réorganisation des filières du système d'enseignement français apparaît comme un véritable problème public (Gusfield, 2009) que ce soit au sein de l'univers politico-administratif (Chapoulie, 2010; Robert, 2015), dans certaines franges du champ scientifique (Chapoulie, 2006), ou encore dans le champ syndical (Dreyfus, 1965; Robert, 2004). Après avoir suscité un grand nombre de projets de réforme restés sans suite (Donegani et Sadoun, 1976, p. 1129), la résolution de ce problème va prendre la forme de trois réformes souvent qualifiées de «structurelles» et désignées par les noms des ministres qui les ont portées: "Berthoin», «Fouchet» et «Haby». S'imposant comme autant de passages obligés de tout discours sur l'histoire du système éducatif, ce sont elles qui ont "surtout retenul'attention des historiens de l'éducation » (D'Enfert et Kahn, 2011, p. 2), occultant dès lors les transformations d'ampleur des programmes et du rapport pédagogique qui ont pourtant accompagné les évolutions morphologiques et institutionnelles du système d'enseignement.

Or, comme l'ont bien montré les recherches entreprises dans le cadre du projet Rédiscol', au cours de la longue période qui s'étend du milieu des années 1940 au milieu des années 1980, «les contenus d'enseignement, les méthodes d'enseignement, les pratiques enseignantes ont [...] connu des mutations, parfois radicales» (D'Enfert et Lebeaume, 2015, p. 10). En effet, on assiste alors à un foisonnement d'initiatives réformatrices : création de grandes commissions, introduction des dernières évolutions de la recherche scientifique dans les programmes scolaires (linguistique et mathématiques modernes), publications de programmes rénovés dans de nombreuses disciplines, création de nouveaux enseignements (activités d'éveil dans le cadre du tiers-temps pédagogique en primaire, technologie et sciences économiques et sociales dans le second degré), etc.

Si la création du CNP ouvre bel et bien une nouvelle période dans l'histoire des réformes des programmes scolaires, ce n'est donc pas, contrairement à ce qu'ont

1 «Réformer les disciplines scolaires: acteurs, contenus, enjeux, dynamiques (années 1950-années 1980)». Initié en 2007 et coordonné par Renaud d'Enfert, ce projet financé par l'Agence nationale de la recherche prend pour objet «les grandes réformes disciplinaires effectuées au cours des années 1960-1970 en les inscrivant dans une dynamique de plus longue durée qui s'origine au tournant des années 1940-1950» (Projet «Rédiscol», rapport final, p. 7 : En ligne <http:// www.ghdso.u-psud.fr/pdf/Rapport_final_REDISCOL.pdf > (consulté parfois pu affirmer certains réformateurs (Gauthier, 1988, 2014; Raulin, 1996, p. 99 ; Raulin, 2006, p. 62) parce que les contenus d'enseignement seraient alors devenus ce qu'ils n'auraient pas été jusqu'alors, à savoir un objet d'intérêt pour le ministère de l'Éducation nationale. La rupture tient d'abord à la temporalité ainsi qu'au cadre politique et institutionnel des transformations des programmes. En effet, jusqu'au début des années 1980, "faute d'une politique d'ensemble, les réformes et projets de réforme des disciplines scolaires, de leurs contenus, de leurs méthodes, se réalisent ou s'élaborent en ordre dispersé. D'une discipline à l'autre, les temporalités et les modalités du processus réformateur, les segments scolaires concernés par celui-ci, l'existence et la nature des commissions de réforme, la qualité de leur président (inspecteur général ou personnalité extérieure à l'administration ministérielle) sont extrêmement variables» ( $D^{\prime}$ Enfert et Kahn, 2011, p. 14)². Or, à l'inverse, la création du CNP va être le levier principal de ce que certains acteurs eux-mêmes vont appeler une «politique des contenus d'enseignement» (Gauthier, 1988), c'est-à-dire une réforme coordonnée, globale et simultanée de l'ensemble des disciplines en fonction de principes communs et applicables de manière transversale. Objectivée institutionnellement non seulement dans le CNP mais aussi dans la création d'un bureau des programmes au sein du ministère de l'Éducation nationale, dans la rédaction d'une "charte des programmes $»^{3}$ ou encore dans la codification d'un nouveau circuit d'écriture des programmes (Clément, 2012), la «politique des contenus d'enseignement» tendrait alors à devenir, à l'instar d'autres «politiques publiques», comme la politique culturelle (Dubois, 1999) ou la politique de la ville (Tissot, 2007), une catégorie d'action publique à part entière, c'est-àdire l'une des «formes stabilisées dans lesquelles l'intervention des pouvoirs publics s'exerce et est donnée à voir» (Dubois, 2003, p. 16).

Cela étant, la création du CNP tend à entériner une seconde rupture, laquelle ne concerne cette fois plus le dispositif institutionnel d'élaboration des programmes, mais les acteurs de ce processus. En effet, la configuration précédente se caractérise par le fait que les inspections générales et les associations de spécia-

2 Dans sa postface au dernier livre collectif issu du projet «Rédiscol» (D’Enfert et Lebeaume, 2015), Jean-Michel Chapoulie conclut dans le même sens et reprend cette même périodisation en écrivant que jusqu'au début des années 1980, "les évolutions de chaque matière enseignée, ainsi que leur temporalité, constituent autant de cas singuliers» (Chapoulie, 2015, p. 241)

3 "Bulletin officiel de l'Éducation nationale, 20 février 1992". 
listes se conduisaient, pour parler comme Joseph Gusfield, comme les véritables «propriétaires » de la question des contenus d'enseignement, c'est-à-dire comme les acteurs possédant l'autorité permettant de désigner une situation donnée comme un problème et de suggérer ce qui doit être fait à son sujet (Gusfield, 1981, p.433). Or, la création du CNP va se traduire, au moins provisoirement ${ }^{4}$, par un transfert de propriété de I'Inspection générale et des associations de spécialiste vers le CNP et, dans une moindre mesure, vers le cabinet du ministre et les directions pédagogiques du ministère (notamment la direction des lycées et collèges).

Afin de mettre au jour les conditions de possibilité de cette double rupture, cet article se propose de revenir sur la pré-histoire du CNP, du début des années 1980 jusqu'à sa création en 1989-1990. ${ }^{5}$ Le début des années 1980 constitue en effet le moment de bascule à partir duquel se dessinent non seulement les linéaments d'un nouveau dispositif institutionnel de réflexion sur les contenus d'enseignement et l'élaboration des programmes, mais aussi le dessaisissement de l'Inspection générale et des associations de spécialistes au profit d'acteurs concurrents. Parce qu'il se donne comme but d'expliquer un tel renversement historique, cet article abordera donc cette période en se focalisant à la fois sur les instruments (Lascoumes \& Le Galès, 2004), en l'occurrence les commissions et les rapports, et sur les acteurs, individuels et collectifs, du changement ${ }^{6}$.

4 Provisoirement, car ce qu'une configuration historique a produit une autre peut le défaire. Ainsi l'arrivée de François Bayrou au ministère de l'Éducation nationale correspondra à un retour dans le jeu de I'Inspection générale.

5 Cette contribution sur la création du Conseil national des programmes associe des éléments issus de la réflexion conduite dans le séminaire d'histoire de l'éducation d'Antoine Prost à Paris I, à d'autres éléments tirés de la thèse de Pierre Clément portant sur la même problématique mais abordée dans une perspective mêlant sociologie et science politique. Les versements AN 19970515 et AN 20070019 ont été les premiers fonds consultés, à la base des deux recherches menées parallèlement sur le CNP. À ces sources se sont ajoutés des témoignages d'acteurs, la consultation des archives privées de Pierre Bourdieu, celles du ministère Savary, AN 20150188 et de la Direction des collèges et lycées, AN 19890113, AN 19910831, AN 20050383. Les archives du Cabinet Jospin AN 20040313 ont été consultées avec dérogation.

6 Si d'autres acteurs, notamment ceux qui ont pu opposer une résistance à ces changements, pourront évidemment apparaître au fil de l'analyse, on ne reconstruira pas ici l'ensemble des positions et prises de position constitutives du champ du pouvoir scolaire, ce qui, de toute façon, excèderait de beaucoup le cadre d'un seul article (Clément, 2013). De même, le télescopage entre les enjeux propres aux transformations des contenus d'enseignement et les enjeux relatifs à la revalorisation financière des enseignants ainsi qu'aux luttes d'appareil et de tendances alors particulièrement vives
Dans un premier temps, on montrera en quoi, d'un côté, l'innovation organisationnelle que représentent les commissions permanentes mises en place par le ministère de l'Éducation nationale et, de l'autre, l'intervention du Collège de France avec la publication des Propositions pour l'enseignement de l'avenir participent d'une stratégie de contournement des intérêts disciplinaires. Dans un deuxième temps, on analysera le travail des commissions de savants, chargés en 19881989 d'une réflexion sur les programmes, à la demande du ministre de l'Éducation nationale, Lionel Jospin, et de son conseiller spécial, Claude Allègre. II apparaitra alors que les contradictions évidentes d'un dispositif oscillant entre autonomie et instrumentalisation de la légitimité scientifique ont pesé sur la naissance du futur CNP.

\section{3-1985 : Dispositifs et acteurs du contournement des intérêts disciplinaires}

Entre 1983 et 1985, le problème des contenus d'enseignement va être essentiellement posé et discuté dans le cadre de deux dispositifs : d'un côté, les commissions permanentes de réflexion sur l'enseignement des disciplines progressivement mises en place sous l'autorité du ministre de l'Éducation nationale Alain Savary à partir du début de l'année 1983 et, de l'autre, la commande, un an plus tard en février 1984, par le président de la République, François Mitterrand, d'une réflexion sur l'enseignement de l'avenir à l'Assemblée des professeurs du Collège de France.

Bien qu'ils portent sur le même problème, ces deux dispositifs obéissent à des logiques politiques différentes et s'inscrivent dans des temporalités et des contextes politiques distincts. Mobilisant les mêmes instruments de gouvernement, à savoir la constitution d'un espace de discussion collective composé de membres officiellement mandatés par le pouvoir politique, ils se distinguent par leur architecture organisationnelle. Au-delà de ces différences, les acteurs impliqués dans chacun d'eux partagent néanmoins un même sens commun réformateur (Topalov, 1999) fondé sur la nécessaire remise en cause de la domination des disciplines dans les processus de changements de programme.

dans le champ syndical (Geay, 2005, p.99) ne sera pas abordé en tant que tel, mais seulement présent en toile de fond. 


\section{Les commissions permanentes de réflexion sur l'enseignement des disciplines : politisation et élargissement du processus d'élaboration des programmes scolaires}

Dès son arrivée au ministère de l'Éducation nationale en mai 1981, Alain Savary s'engage dans une vaste tentative de transformation du système éducatif, ainsi qu'il l'explique dans un bilan dressé après sa démission de juillet 1984 : «Deux objectifs s'imposaient : faire évoluer le système éducatif dans le sens d'une plus grande démocratie et améliorer la qualité de ses prestations [...]. La lutte contre l'échec scolaire et les inégalités sociales qui lui sont liées, la redéfinition et la modernisation des contenus d'enseignement, la formation des enseignants, la décentralisation et l'autonomie des établissements s'inscrivaient dans cette double exigence ${ }^{7}$. Pour nourrir la réflexion, il met en place plusieurs commissions qui rendront chacune un rapport (Prost pour les lycées, Legrand pour les collèges, Soubré pour la décentralisation, etc.). Aucune ne concernait dans un premier temps les contenus d'enseignement. Ce n'est qu'en mars 1983 que des commissions permanentes de réflexion sur l'enseignement des disciplines (COPREX) sont créées, pilotées jusqu'en juillet 1984 depuis le cabinet du ministre, en particulier par Catherine Moisan ${ }^{8}$, chargée du «suivi pédagogique et [de] la conduite des travaux des différentes commissions ${ }^{9}$, ce qui prouve l'importance politique accordée au dossier.

La décision a été annoncée le $1^{\text {er }}$ février 1983 lors d'une conférence de presse du ministre. Un nouveau mode d'élaboration des contenus d'enseignement, la cinquième orientation, est alors présenté par Alain Savary: «Il faut substituer aux rivalités dont les disciplines trop cloisonnées sont aujourd'hui le théâtre, une perspective plus globale de formation [...] II faut en outre définir un cadre national à partir duquel les adaptations locales que je crois fort nécessaires peuvent se faire. J'envisage

7 SAVARY A. (1985), En toute liberté, Paris, Hachette, p. 53.

8 Recrutée au cabinet d'Alain Savary en février 1983, Catherine Moisan, ancienne de l'École normale supérieure de Sèvres et professeur agrégée de mathématiques, s'est vue confier trois missions liées à la rénovation : les zones d'éducation prioritaires, le dossier immigration et la pédagogie interculturelle, la révision des contenus d'enseignement. Elle est officiellement en charge du dossier, mais Thérèse Delpech et Yvon Robert travaillent aussi sur ce sujet. Entretien du $1^{\text {er }}$ juillet 2015 avec Catherine Moisan.

9 C'est en ces termes qu'Alain Savary termine sa lettre de mission dès la fin de ce mois la création de commissions permanentes qui vont réexaminer les contenus d'enseignement avec le souci de déterminer des objectifs, qui pourraient être atteints par différentes voies, à différents rythmes, de penser les relations entre les différentes matières et de donner aux élèves des connaissances peut-être moins nombreuses mais plus solides, mieux articulées et plus méthodiquement présentées ${ }^{10}$. Ce qui induit que, désormais, les logiques propres aux différentes disciplines ne seront plus premières dans l'évolution des programmes, alors même que ces programmes restent les garants de l'unité d'un système éducatif que le ministre aspire à diversifier en prônant davantage d'autonomie à tous les niveaux.

Une note d'information"1 datée du 4 mars, fournit le cadre et les objectifs, précisant les missions des deux types de commissions mises en place : horizontales par niveau d'enseignement; verticales par discipline ou groupes de disciplines, portant sur l'ensemble des cycles. Composées d'inspecteurs, d'universitaires, de chercheurs, d'enseignants du premier et du second degré, parfois de représentants des professions, celles-ci comprennent au moins "une ou deux personnes spécialistes de domaines voisins » (p. 2) sur leurs vingt à trente membres. Chaque direction du ministère a un représentant permanent en "qualité d'observateur» (ibid.). Les membres sont nommés pour un mandat de deux ans renouvelable.

Dès le printemps 1983, dans un courrier du ministre à chaque président de commission verticale, il est précisé que leur compétence "porte sur l'ensemble des formations (de la maternelle à l'université) [et comprends] une mission d'évaluation (interne et externe) et de prospective $»^{12}$. Mais les commissions verticales, ainsi qu'il sera énoncé quelques mois plus tard, doivent aussi "réfléchir aux liens nécessaires entre la rénovation des contenus d'enseignement et les activités éducatives ainsi qu'à la formation des enseignants et à la recherche pédagogique »13.

10 Conférence de presse sur les collèges, 1er février 1983, AN 20150188/75-1-76 et En ligne <http://discours.vie-publique.fr/ notices/833052700.html> (consulté le 20 septembre 2017)

11 Note d'information du 4 mars 1983 «Réexamen des contenus d'enseignement dans les écoles, collèges et lycées», p. 1. AN 19910831/9

12 Direction des lycées. «Rapport sur la création de commissions permanentes de réflexion sur l'enseignement des disciplines (COPREX)», p. 3. AN 19890113/6

13 Note d'information du 5 octobre 1983 «Réexamen des contenus d'enseignement dans les écoles, collèges et lycées», p. 2. AN $19910831 / 9$ 
Sont concernés dans un premier temps les mathématiques, la biologie et la géologie, le français, la technologie. Les trois premières commissions sont présidées par des universitaires, respectivement Jean Martinet, professeur de mathématiques à Strasbourg, Luc Picon, professeur de physiologie à Paris 7, Jean-Claude Chevalier, professeur de linguistique à Paris 8 ; seule la technologie est présidée par un inspecteur général, grande figure de l'enseignement technique, Lucien Géminard. Une cinquième commission verticale est créée en septembre 1983, celle d'histoire et de géographie, confiée à Jacques Le Goff, directeur d'études à l'école des hautes études en sciences sociales (EHESS) ${ }^{14}$. Enfin, les deux dernières le seront à l'automne : celle de physique sous la direction d'André Guinier, président de l'université Paris XI et auteur pour I'UNESCO en 1981 d'un rapport sur l'enseignement de la physique en Tunisie; celle d'éducation physique et sportive sous la direction d'Alain Hébrard alors chargé de cours à l'université de Montpellier avant d'y être nommé maitre de conférences l'année suivante.

Le choix de confier la quasi-totalité des présidences des commissions verticales à des universitaires marque bien le souhait d'Alain Savary d'élargir la composition de ces instances à des personnalités scientifiques reconnues et extérieures à l'administration ministérielle.

Cet élargissement se manifeste également par la présence des directions pédagogiques (écoles, collèges, lycées) tant dans les commissions verticales avec trois représentants permanents, un par direction, que dans chaque commission horizontale où la présidence est assurée par le directeur pédagogique concerné. Cependant, le cabinet a bien veillé à n'exclure personne et chaque commission regroupe "l'inspection générale, les principaux partenaires (syndicats, fédérations de parents d'élèves, représentants de la conférence des associations de spécialistes), des membres de I'INRP [Institut national de recherche pédagogique] et des personnalités choisies en fonction de leurs compétences » ${ }^{15}$.

La commission horizontale «collèges » est en place au printemps 1983, celles des écoles et des lycées (cycle long et court) à l'automne alors qu'une seconde note d'information au même libellé, «Réexamens des contenus d'enseignement dans les écoles, collèges et lycées ", précise certains points et synthétise les objectifs et missions des deux types de commissions :

14 Lettre de mission du 27 septembre 1983 AN 19910831/9.

15 Note d'information du 4 mars 1983, op. cité. p. 1.
«1. Assurer une plus grande cohérence entre les différents niveaux d'enseignement aujourd'hui trop cloisonnés [...] 2. Mieux articuler entre elles les différentes disciplines qui doivent être pensées dans leurs relations mutuelles. [...] 3. Permettre une adaptation permanente à l'évolution des connaissances et des techniques. [...] 4. Définir un cadre national dans lequel s'exercera une plus grande responsabilité des établissements. [...]» ${ }^{16}$. Les commissions horizontales doivent formuler de plus «un avis sur la cohérence des commissions verticales ainsi que sur les instructions officielles à élaborer $\gg^{17}$.

En témoin, Claude Pair, alors directeur des lycées, raconte le fonctionnement collégial de ce temps-là. « $A$ cette réflexion participaient les directeurs des directions pédagogiques, dont j'étais. Les directions étaient totalement associées et, contrairement à ce quej'ai pu connaître ensuite, il n'y avait pas à cette époque d'un côté le cabinet et de l'autre les directions. Pour ce qui concerne la mienne, nous avions en particulier beaucoup travaillé sur les organes pouvant conseiller le ministre sur la définition des contenus d'enseignement, ce qu'a fait à partir de 1990 le Conseil national des programmes [...]. Nous étions partis dans une autre direction, fondée sur l'expérience des Commissions professionnelles consultatives, et parvenus à la conclusion qu'il fallait confier cette tâche à deux sortes de commissions permanentes associant l'administration, les corps d'inspection, les partenaires syndicaux : les unes, dites "horizontales", transversales aux disciplines, organisées par niveau d'enseignement, en particulier pour fixer les objectifs généraux de chaque filière de formation; les autres, dites verticales, par disciplines, pour fixer les objectifs détaillés et les programmes, avec en outre la participation de personnalités qualifiées. Cette proposition avait été mise au point avec le groupe sur les lycées présidé par Antoine Prost ${ }^{18}$ [...]. Avec l'approbation du ministre, nous avons dès 1983 mis en place deux commissions horizontales, l'une pour les lycées d'enseignement général et technique, l'autre pour les lycées d'enseignement professionnel. Nous avons aussi mené en 1982-1983 une réflexion avec la direction compétente, et sa directrice Danièle Blondel, pour définir les "prérequis" pour l'entrée

\footnotetext{
16 Note d'information du 5 octobre 1983, op. cité, p. 1.

17 Ibid. p. 2.

18 Le groupe de travail national sur les seconds cycles présidé par Antoine Prost a été créé fin 1981 à l'initiative de Claude Pair, afin de «susciter et de promouvoir la réflexion et l'innovation dans les lycées» (Prost, 1983, p. 7). En liaison permanente avec la Direction, il a rendu son rapport en novembre 1983. Il est favorable à la création des commissions verticales car «Une réflexion permanente est nécessaire sur les disciplines, comme sur les formations» (ibid. p. 163).
} 
dans les diverses filières de l'enseignement supérieur. $»^{19}$

Claude Pair consacre un chapitre à la question des contenus d'enseignement dans l'ouvrage qu'il publie après son départ du ministère (Pair, 1986). Il revient sur le travail des commissions en conclusion de son analyse critique sur les modalités d'élaboration traditionnelles, et il prône la création au ministère d'un «bureau d'études spécialisé dans la définition des contenus», qui travaillerait en lien avec « des commissions formées de personnalités compétentes, représentatives et d'origine variée» (p.102). Car pour lui, «Apporter davantage de clarté dans les contenus de l'enseignement est une exigence de la démocratie» (p. 110). S'il a bien été associé à la réflexion, il n'a pas eu le temps de mettre en œuvre cette nouvelle organisation qui ne verra le jour qu'au début des années 1990. La Direction des lycées et collèges se saisira alors du dossier, avec l'accord d'André Legrand, son directeur.

Les dispositifs mis en place en 1983 innovent et introduisent une nouvelle organisation qui préfigure I'institution CNP. D'abord, une même temporalité régit désormais toute révision des programmes de toutes les disciplines; ensuite une coordination permanente associe le cabinet et les directions pédagogiques dans ce processus, auquel participent également des représentants des syndicats ainsi que les acteurs antérieurs, inspecteurs généraux et représentants des associations disciplinaires. L'élargissement effectif de commissions pérennes, puisque devant fonctionner dans la durée, couplé à cette temporalité commune, la présence d'universitaires reconnus pour leurs compétences, constitue bien une innovation institutionnelle et organisationnelle qui se concrétisera en 1990 avec la création du CNP.

Alain Savary démissionne le 16 juillet 1984 et son testament politique sera consacré justement à la question des contenus d'enseignement. Publié en novembre 1984 par Le Monde de l'éducation (p.54-58) quatre mois après la démission du ministre, ce document d'importance est une vraie feuille de route, au contenu politique affirmé. Rédigé par Catherine Moi$\operatorname{san}^{20}$ et diffusé par les services ministériels immédiatement après le départ du ministre, il montre «com-

19 Lettre de Claude Pair, du 2 septembre 2015, en réponse à nos questions.

20 «C'est Catherine Moisan qui avait préparé le texte qui a conduit au "testament", [...] et mis au point dans une réunion à laquelle je participais, réunion irréaliste puisque le Ministre avait déjà envoyé sa lettre de démission; il ne nous l'avait pas dit, mais nous nous en ment la réflexion sur les contenus d'enseignement est indissociable de celle sur les structures ${ }^{21}$.

Prévu comme une note d'information datée du 18 juillet 1984, ce document comprenait une lettre de deux pages signée de Savary accompagnée d'un texte en trois parties, adressé à tous les partenaires, organisations syndicales et associations de parents d'élèves ${ }^{22}$. Le courrier, forme de bilan de son action au ministère, rappel de «l'effort de rénovation d'ensemble du système éducatif [...] engagé depuis trois ans» (p. 1), met en avant trois axes, dont le second est formulé ainsi : «faire évoluernotre enseignement en vue du nécessaire développement scientifique, technique, économique et social de notre pays. II s'agit, bien sûr, de la modernisation indispensable des connaissances et des compétences qu'apporte la formation à nos élèves » (p. 2). Il est rappelé que «les modifications successives apportées ont toujours été partielles : elles concernaient tantôt les structures, tantôt les programmes de tel ou tel niveau dans telle ou telle discipline». Le ministre conclut : "c'est l'ensemble des contenus qui doit être repensé, en fonction de principes et d'objectifs clairement définis ». Suivent alors, dans une première partie, six orientations générales pour cette rénovation des contenus qu'il souhaite voir débattue. La seconde partie présente les orientations spécifiques par niveau, écoles, collèges, lycées ${ }^{23}$; la troisième, plus courte, concerne les textes officiels.

Le CNP, dans la charte des programmes notamment, reprendra à son compte un certain nombre d'éléments énoncés dans ce texte de 1984. Ainsi, I'inversion de la hiérarchie des normes pédagogiques : les objectifs généraux de formation sont premiers, les contenus disciplinaires viennent ensuite, ce qui est un vrai renversement de perspective; ou bien l'apparition de la notion de compétences.

21 Introduction du Monde de l'éducation à ce "document, inédit», p. 54.

22 AN 20150188/75-1-76

23 Nourries du travail des commissions horizontales, les propositions par niveau contiennent nombre d'éléments qui verront le jour les années suivantes : «objectif d'amener, d'ici dix ans, $80 \%$ d'une classe d'âge à la fin du second cycle long ou court», proposition reprise du rapport Prost (1983, p.66); cycles à l'école primaire; au collège, définir des niveaux d'exigence "sous forme de paliers jalonnant la progression des élèves». 
1. Repenser l'enseignement de chaque discipline, non pas pour elle-même, mais en fonction des finalités globales de la formation. [...] II fait donc faire cesser l'éternel débat posé en termes de concurrence et de priorité, et, en faire surgir un nouveau en termes d'équilibre des contenus et de cohérence. [...]

2. Amener les élèves à une réelle maîtrise des connaissances. Le mot connaissance est pris ici au sens large, imbriquant, sans les dissocier, savoirs et savoir-faire spécifiques à chaque domaine d'enseignement. [...] il faut être particulièrement attentif à une véritable continuité pédagogique afin d'atténuer les ruptures dans le cursus scolaire. Dans certains domaines, il s'agit de préciser ces connaissances sous forme d'objectifs, sans oublier les niveaux d'exigence. [...] Dans d'autres domaines, il s'agit d'alléger la somme des connaissances définies actuellement dans les programmes. C'est la seule solution pour être vraiment exigeant et rigoureux dans la formation de tous les élèves. [...]

3. Développer le travail personnel des élèves. [...] repenser les contenus à tous les niveaux, non seulement sous l'angle de l'enseignement que le maître doit dispenser, mais aussi sous l'angle des travaux et des activités que les élèves doivent effectuer de façon progressivement autonome. [...] II faut donc absolument que, dès l'école élémentaire, les résultats obtenus (notes, compréhension, réalisation, etc.) soient fortement et explicitement reliés à l'activité personnelle de l'élève.

4. Prendre en compte, dans l'enseignement de chaque discipline, le développement de compétences transversales chez les élèves. [...] II ne s'agit pas de faire de ces compétences des objets d'enseignements spécifiques, coupés des contenus disciplinaires; mais il est nécessaire d'expliciter, d'une part, en quoi chaque discipline participe au développement de telle ou telle de ces capacités transversales et, d'autre part, les objectifs et les niveaux d'exigence pour chaque cycle. [...]

5. Relier les méthodes pédagogiques aux contenus d'enseignement et tenir compte de leurs conséquences sur la formation des élèves. [...]

6. Définir le cadre national qui permette une réelle autonomie des établissements ${ }^{b}[\ldots]$

a : Les extraits soulignés le sont dans le texte original.

b : Cette orientation est absente dans l'article du Monde de l'éducation, sans que l'on puisse en préciser la raison.

Le départ d'Alain Savary et de son équipe ne réduit pas à néant ce qui avait été amorcé. Ainsi, la commission horizontale «collèges» continue son travail, c'est toujours le Directeur des collèges, en l'occurrence André Hussenet, qui la préside sur la période 1984-1986²4. La réflexion entreprise entre 1983 et 1984 à l'initiative du cabinet et des directions pédagogiques ne disparaît pas complètement ${ }^{25}$ et certains acteurs, tel Jean-Claude Chevalier, œuvreront à nouveau quelques années plus tard, mais dans un tout autre contexte.

En parallèle, mais sans lien aucun, l'Élysée se penche sur la question des contenus d'enseignement, sans consulter, manifestement, Alain Savary et son cabinet $^{26}$. Cette juxtaposition des démarches est pour

\section{AN 20050383/1}

25 En mai 1985, le nouveau ministre adresse une lettre au doyen de l'Inspection générale insistant sur la nécessité «d'identifier ce qui est indispensable au collégien d'aujourd'hui à l'issue de la classe de $3^{\mathrm{e}}$ » ce qui «exige que soient clairement connus les objectifs généraux du collège ». Il s'agit « de permettre l'élaboration des nouveaux programmes sur des bases claires». Lettre du ministre de I'Éducation nationale Jean-Pierre Chevènement au doyen de l'Inspection générale de l'Éducation nationale, mai 1985. Archives du SNI-PEGC, boîte $n^{\circ} 1787$

26 «Je ne me rappelle pas qu'Alain Savary m'en ait parlé, ni son successeur Jean-Pierre Chevènement. [...] Alain Savary n'a pas été associé à cette réflexion. » Claude Pair, ibid.

Situation confirmée par Michèle Gendreau-Massaloux dans un partie liée aux difficiles relations et au contentieux persistant entre le ministre et le Président depuis le congrès d'Epinay mais aussi certainement à leurs parcours et leurs engagements politiques très différenciés, tant pendant la Seconde Guerre mondiale que pendant la Guerre d'Algérie. Elle n'en reste pas moins surprenante.

\section{Les Propositions pour l'enseignement de l'avenir : les professeurs du Collège de France contre le corporatisme de discipline}

Le 13 février 1984, le président de la République François Mitterrand adresse une lettre de mission à l'administrateur du Collège de France, Yves Laporte, dans laquelle il lui demande que le Collège «veuille bien réfléchir à ce que pourraient être, selon lui, les principes fondamentaux de l'enseignement de l'avenir ${ }^{27}$. Comment expliquer ce recours littéralement extraordinaire au

courrier du 31 août 2015 : «Le ministre ne voyait pas le Président sur ces questions». Conseillère technique à l'éducation en mars 1984, elle devient secrétaire générale adjointe de la Présidence de la République de 1985 à mai 1988.

27 Lettre de François Mitterrand à l'Administrateur du Collège de France, 13 février 1984, in Collège de France, Propositions pour l'enseignement de l'avenir, Paris, Presses du Palais Royal, 1985, p. 5. 
Collège de France, tant il est rare que celui-ci, en tant qu'institution, ou que l'Assemblée des professeurs, à titre collectif, prenne position sur un problème public ? ${ }^{28}$ Pour ce faire, il faut rappeler qu'au moment où germe l'idée d'un tel rapport, c'est-à-dire entre décembre 1983 et janvier 1984, la situation politique du gouvernement se trouve considérablement affaiblie par la conjonction de plusieurs mouvements de protestation portant, justement, sur la politique scolaire menée par le ministre de l'Éducation nationale Alain Savary : le premier s'en prend violemment au projet de nationalisation de l'enseignement privé, le second dénonce une supposée dégradation de la qualité de l'enseignement, le troisième se focalise sur "l'affaire de la qualité des universitaires» (Lelièvre et Nique, 1995).

S'agissant de ce nouvel épisode de la «guerre scolaire» (Mayeur, 1985), le mois de décembre 1983 voit ainsi les premières vagues de grandes manifestations et contre-manifestations qui ne s'arrêteront plus jusqu'à atteindre leurs points culminants les 4 mars, 25 avril et 24 juin 1984. C'est également le moment où le conflit se déplace dans le champ politique. Si les attaques de la droite contre Alain Savary n'ont jamais cessé, le ministre doit également faire face à une opposition de plus en plus vive dans son propre camp. Alors que commence la séquence politique qui aboutira au retrait du projet de loi et à la démission d'Alain Savary en juillet 1984, le président de la République estime d'ores et déjà qu'une solution de compromis est impossible (Lelièvre et Nique, 1995, p. 280). Face à ce qui s'annonce comme une des plus grandes défaites de la gauche sur la question scolaire et face à l'épuisement de ce thème traditionnellement fédérateur qu'a été jusqu'alors la laïcité, l'idée de faire appel à cette autorité incontestée que représente le Collège de France pour essayer de sortir de l'ornière où, à tout le moins, de tracer les voies d'une politique progressiste peut apparaître comme un recours possible pour le président de la République.

Ce recours au Collège de France se comprend d'autant mieux si l'on sait que ce nouvel épisode de la guerre scolaire se conjugue alors avec une série de critiques sur la qualité de l'enseignement public. Depuis

28 Peu de temps avant, en 1979, un rapport sur l'avenir de la biologie, intitulé Sciences de la vie et société, avait bien été remis au président de la République Valéry Giscard d'Estaing par deux professeurs du Collège, François Gros et François Jacob en collaboration avec Pierre Royer, pédiatre, professeur de médecine et chef de département à I'hôpital Necker. Mais, contrairement au rapport de la fin de 1982, l'idée que l'école est en «crise» commence à s'imposer dans l'espace public (Balland, 2009). De nombreuses voix s'élèvent en effet pour dénoncer les conséquences catastrophiques du collège unique. En 1982 et 1983, les projets de rénovation du collège radicalisent les positions. À l'occasion de la parution de pamphlets ou de livres polémiques, le champ médiatique commence à cette période à être parcouru de tribunes et de prises de position qui dénoncent la dégradation et le déclin inexorable de l'enseignement (Balland, 2009, p. 142-179). Tendant à s'imposer comme un allant de soi, la rhétorique de la crise ne laisse pas François Mitterrand indifférent. C'est ainsi que lors du conseil des ministres du 31 août 1983, il fait part de sa vive inquiétude s'agissant de l'état de l'enseignement de I'histoire. II formule également des observations critiques sur le projet de suppression des mentions au bac ainsi que sur l'état de l'enseignement de l'informatique et de la philosophie. Ce sont là autant de jugements qui constituent un désaveu pour son ministre de l'Éducation nationale. Le 2 septembre, la colère présidentielle s'affiche en une du Monde qui publie un article intitulé «Le coup de sang de Mitterrand».

Les universités ne sont pas non plus épargnées par les mobilisations. Comme le rappellent Claude Lelièvre et Christian Nique, «la question de l'enseignement supérieur est l'une des plus préoccupantes et des plus difficiles pour le premier gouvernement de François Mitterrand» (Lelièvre et Nique, 1995, p. 305). Défendue par certains partis et syndicats marqués à gauche, la création d'un corps unique regroupant les enseignants-chercheurs de rang $A$ et de rang $B$ suscite en effet une opposition virulente chez certains universitaires qui voient dans ce projet une attaque contre le mérite et la qualité de la science. Menée par le mathématicien Laurent Schwartz ${ }^{29}$ qui a l'oreille de François Mitterrand, la fronde aboutit à ce que le président de la République prenne position non seulement contre le corps unique, mais aussi contre la suppression des agrégations du supérieur envisagée par Alain Savary. Bien que ce conflit se solde finalement par une victoire des parti-

29 Frère de Bertrand Schwartz, Laurent Schwartz a été chargé du rapport consacré à l'enseignement et au développement scientifique (Schwartz, 1981) dans le cadre de la commission du bilan mise en place par Pierre Mauroy après l'élection par François Mitterrand et présidée par François Bloch-Lainé (Schwartz, 1997). Il y dresse un constat très sévère sur l'état du système d'enseignement français dénonçant notamment l'absence de sélection. Du mouvement contre la politique universitaire d'Alain Savary naît l'association "Qualité de la science française » encore active aujourd'hui. 
sans de "l'excellence » et de la «qualité de la science», François Mitterrand craint néanmoins qu'il n'ait laissé des traces sur son image auprès des intellectuels et des scientifiques.

Dans ce contexte politiquement très délicat pour le pouvoir en place, I'appel au Collège de France, c'està-dire à une institution placée au sommet de la hiérarchie intellectuelle et scientifique, apparaît non seulement comme le moyen de réaffirmer le lien privilégié entre le président de la République et les intellectuels, mais aussi comme une manière de sortir de cette crise multiforme par le haut, c'est-à-dire sans revenir à l'ordre ancien et sans renoncer à toute velléité de transformation du système d'enseignement.

Si le texte de 48 pages qui sera finalement remis au président de la République va bien au-delà de cette idée d'une nécessaire révision des contenus d'enseignement, ce principe apparaît néanmoins comme un aspect essentiel du texte. Ainsi figure-t-il dès la première version du texte rédigée en avril 1984 «à la hâte $»^{30}$ par Pierre Bourdieu pour servir de point de départ à la réflexion collective des professeurs du Collège de France. Face à "l'inertie structurale du système d'enseignement qui se traduit par un retard, variable selon les moments et les disciplines, des contenus enseignés par rapport aux acquis de la recherche», cette première version pose comme premier principe «la mise en question permanente de la culture enseignée $»^{31}$. II s'agit à la fois de rénover régulièrement les contenus d'enseignement pour éviter leur obsolescence, mais d'ouvrir leur processus d'élaboration afin d'«éviter que le système scolaire ne se constitue en univers séparé, sacré, proposant une culture elle-même sacrée et coupée de l'existence ordinaire $»^{32}$.

Dans cette première version, l'idée de créer une institution spécifiquement dédiée à la mise en œuvre n'apparaît cependant pas. C'est lors d'une première réunion formelle, organisée le 19 octobre 1984 par le Collège de France avec les professeurs volontaires, qu'il en est fait mention. S'appuyant explicitement sur les prises de position de certains de ses collègues, dont Pierre-Gilles de Gennes qui expliquait que s'agissant des programmes "un besoin premier est d'élaguer ${ }^{33}$,

30 Lettre de Pierre Bourdieu à Henri Dieuzeide, 25 juin 1984. Archives personnelles de P. Bourdieu.

31 Version d'avril 1984 du rapport du Collège de France. Archives personnelles de P. Bourdieu.

32 Ibid.

33 Lettre de Pierre-Gilles de Gennes à Pierre Bourdieu, 27 juin 1984. Archives personnelles de P. Bourdieu.
Pierre Bourdieu propose «la création d'une instance permanente de révision des savoirs enseignés ${ }^{34}$. Recueillant un large assentiment parmi les professeurs du Collège, cette proposition sera reprise telle quelle dans la version finale des Propositions. Parmi les neuf principes qui y sont énoncés, le cinquième porte en effet sur «la révision périodique des savoirs enseignés». Pour aller à l'encontre de l'inertie structurale liée au «corporatisme de discipline », à la "tendance à l'encyclopédisme» ainsi qu'à "l'institution de préalables donnés pour préréquisits absolus ("on ne peut pas ignorer ça") », le Collège de France propose de "réunir un comité de révision des programmes (des enseignements fondamentaux et secondaires) chargé d'élaguer les programmes de toutes les exigences vestigiales et d'introduire le plus rapidement possible les innovations utiles $\|^{35}$. Mieux, les professeurs du Collège de France vont plus loin en suggérant que ce comité «devrait avoir le pouvoir d'orienter la politique d'investissements publics en matière d'éducation, en définissant les secteurs à réduire ou à augmenter $»^{36}$.

Remises au président de la République, le 27 mars 1985, les Propositions pour l'enseignement de l'avenir vont rencontrer un écho important et largement positif dans la presse écrite ainsi qu'à la télévision, par exemple auprès de Bernard Pivot qui, le 10 mai 1985, invite Pierre Bourdieu à son émission Apostrophes pour présenter le travail du Collège de France en présence, notamment, du ministre de l'Éducation nationale JeanPierre Chevènement. Malgré cela, les Propositions vont pourtant rester, en tout cas dans l'immédiat, quasiment lettre morte. Ainsi, dès le mois d'août 1985, Pierre Bourdieu constate que "pour le moment, le gouvernement ne s'est pas vraiment saisi de ce qui, étant donné l'autorité symbolique du Collège, aurait pu être un instrument considérable de transformation $\Perp^{37}$.

Destinées à sortir par le haut d'une situation de crise multiforme qui, dans l'intervalle, s'est en grande partie dissipée, mais aussi à faire contrepoids à la politique d'Alain Savary qui, entre-temps, a démissionné, les Propositions ont en effet perdu une partie de leur raison d'être politique au moment où elles sont rendues publiques. Leur critique du corporatisme des disciplines ne rencontre par ailleurs que peu d'écho

\footnotetext{
34 PV de la réunion du 19 octobre 1984. Archives personnelles de P. Bourdieu.

35 Collège de France, Propositions pour l'enseignement de l'avenir, Paris, Presses du Palais Royal, 1985, p. 31.

36 Ibid.

37 "Le rapport du Collège de France. Pierre Bourdieu s'explique»,
} La Quinzaine littéraire, 1er août 1985. 
chez Jean-Pierre Chevènement, lequel met en scène sa rupture avec l'entreprise de rénovation pédagogique de son prédécesseur. Tout en saluant la qualité et l'utilité du travail des professeurs du Collège de France, François Mitterrand ne retient ainsi que trois des neuf principes qu'ils ont énoncés dans les Propositions. Si «la nécessité de soumettre les programmes à une révision périodique, destinée à opérer les allègements et les renouvellements indispensables ${ }^{38}$ en fait bel et bien partie, le président de la République renonce toutefois à créer une instance chargée de mettre ce principe en application, se contentant d'annoncer deux nouvelles mesures : la création d'une université ouverte et celle d'une chaîne de télévision éducative et culturelle ${ }^{39}$.

Bien que les Propositions pour l'enseignement de l'avenir ne débouchent pas sur la création immédiate d'un comité de révision des programmes, elles vont néanmoins contribuer à légitimer l'idée de la nécessité d'une telle instance pour réformer les programmes. Un signe de l'appropriation de cette idée par l'aile réformiste de l'administration centrale de l'Éducation nationale apparait dans le livre que Roger-François Gauthier, alors chef du bureau des enseignements généraux et technologiques au sein de la direction des Lycées, entreprend d'écrire en 1986. Développant une critique virulente des corporatismes disciplinaires et de l'Inspection générale, ce livre finalement publié en février 1988 constitue en effet un plaidoyer en faveur d'«une remise en cause profonde [...] de l'ensemble des structures qui participent à l'élaboration des contenus d'enseignement » qui passerait par la création de "commissions nationales consultatives sur les contenus d'enseignement » permettant d'«arracher les contenus d'enseignement au microcosme scolaire» (Gauthier, 1988, p. 131-135) ${ }^{42}$.

Commele montre cet extrait du livre de Roger-François Gauthier, les commissions permanentes mises en place et pilotées par le cabinet d'Alain Savary ainsi que les Propositions pour l'enseignement de l'avenir ont donc laissé des traces non négligeables dans le champ politico-administratif : elles ont permis de poser autrement le problème des contenus d'enseignement et

38 Allocution prononcée par François Mitterrand au Collège de France, Archives nationales, sous-fonds de Michèle Gendreau-Massaloux, conseiller technique pour l'éducation nationale à la présidence de la République de mars 1984 à juillet 1985, AG/5(4)/ MGM 43.

39 Sur le rôle de Georges Duby, Pierre Bourdieu et des Propositions dans I'histoire de la création de cette chaîne qui deviendra la SEPT puis ARTE, voir le témoignage de Jean-Michel Meurice (Meurice, 2011) également disponible sur le site du magazine Télérama. En d'envisager de nouvelles manières de le résoudre. Audelà de leurs différences, l'efficacité relative de ces deux instruments s'explique finalement par leur approche systémique et globale des contenus, par leur ouverture à des acteurs d'ordinaire exclus du débat sur les programmes, par la mobilisation de la légitimité scientifique, laquelle vient concurrencer la légitimité pédagogique et disciplinaire de l'Inspection générale et des associations de spécialistes et, enfin, par un soutien politique fort, du ministère d'un côté, de la présidence de la République de l'autre. Reposant sur ces quatre piliers, cette efficacité ne résistera toutefois pas à l'effondrement du dernier d'entre eux qu'entraîne le retournement de la conjoncture politique advenant avec la démission d'Alain Savary et l'arrivée de JeanPierre Chevènement. Ce n'est qu'en 1988 avec l'arrivée de Lionel Jospin et de Claude Allègre, enseignant chercheur en physique, alors professeur à l'université Paris VII, rue de Grenelle que le projet réformateur dessiné par les commissions et le Collège de France retrouvera un véritable soutien politique.

\section{8-1989 : des commissions de savants sous contraintes budgétaire et politique}

Ancien premier secrétaire du parti socialiste, Lionel Jospin est très peu au fait des questions éducatives lorsqu'il est nommé à l'Éducation nationale en mai 1988. C'était le secrétaire national à l'éducation, Laurent Fabius, qui, en amont des présidentielles, avait travaillé avec la FEN, principal syndicat enseignant, dont la tendance majoritaire constitue alors un vivier de voix socialistes. Lionel Jospin refuse d'emblée le projet imaginé sur la base «pas de revalorisation sans contreparties ${ }^{40}$, conseillé en cela par son ami et conseiller spécial Claude Allègre, qui va s'investir fortement pour élaborer un projet alternatif. Des désaccords avec Michel Rocard, Premier ministre du second septennat de François Mitterrand, vont par ailleurs entacher la première année de fonctionnement : tant sur le fond que sur la méthode, son interventionnisme public dans le domaine éducatif est mal vécu par le ministre qui

40 Ce qu'il annoncera clairement à ses interlocuteurs lors de la table ronde sur la revalorisation de la fonction enseignante le 18 janvier 1989: "Je ne viens pas vers vous dans un esprit de "donnantdonnant", ou dans l'optique d'un troc». AN 20040313/2 
réagit fermement ${ }^{41}$. Alors que les négociations sur la revalorisation de la fonction enseignante interfèrent avec le projet de loi d'orientation, le ministre devant mener les deux de front, la question des programmes ajoute un motif supplémentaire de désaccord, en particulier avec le SNES ${ }^{42}$.

Le processus de création du CNP aboutit en février 1990 avec la parution du décret ${ }^{43}$ mais toute la période est marquée par des tensions fortes entre l'ensemble des protagonistes, ce qui va nuire à sa légitimité et obérer ses premières années de fonctionnement.

\section{Claude Allègre et ses pairs : entre autonomie et instrumentalisation ${ }^{44}$}

Dès juin 1988, des séminaires portant sur les enseignements scolaires et supérieurs réunissent les principaux membres du cabinet ${ }^{45}$. Pour le séminaire du 24 juin ${ }^{46}$, deux notes, rédigées en amont et annotées du ministre, préparent le travail collectif : celle de Paul Rollin, "L'éducation pour tous : les nouveaux défis pour le système éducatif » et celle de Claude Allègre «Quelques suggestions pour l'enseignement primaire (première ébauche)». Le conseiller spécial insiste sur la priorité de revaloriser les instituteurs (traitements et carrière) ${ }^{47}$, mais il estime que "la revalorisation ne fera pas tout», car il faut aussi réorganiser le temps scolaire et périscolaire, et revoir les contenus des programmes: "l'école doit être le lieu d'où l'on sort en maîtrisant la trilogie lire, écrire, compter. [...] on devra généraliser la pédagogie glissante, c'est-à-dire le non-redoublement (jusqu'au cours moyen $2^{e}$ année) mais avec des séances de rattrapage [...] Toute la pédagogie glissante doit être orientée

41 Ainsi dans un courrier manuscrit du 3 janvier 1989 qu'il envoie à Michel Rocard, suite à une interview du Premier ministre au Monde de l'éducation. AN 20040313/1

42 Principal syndicat du secondaire, le SNES se positionne plus à gauche sur l'échiquier syndical que la FEN à laquelle il appartient encore.

43 Le décret 90-179 met en œuvre l'article 6 de la loi d'orientation sur l'éducation du 10 juillet 1989, dite loi Jospin.

44 Les éléments rapportés dans cette partie sont extraits des archives du cabinet Jospin classées en AN 20040313/1 et 20040313/2, consultables seulement avec dérogation.

45 Participent à ces séminaires le directeur de cabinet Olivier Schrameck et plusieurs conseillers techniques, dont Jean Geoffroy, Luc Soubré, Jean Ferrier, Pierre Trincal, Paul Rollin et Daniel Bancel. 46 Toutes les citations de ce paragraphe sont extraites des notes manuscrites du ministre. AN 20040313/1

47 Propos qu'il confirme lors du séminaire : "La revalorisation, c'est le primaire d'abord. Je ne suis pas pour le "donnant-donnant", en tout cas pas pour le "travaillez plus, on revalorisera" ». Notes de Lionel Jospin, p. 11. vers ce but unique, maîtriser la lecture, le calcul, l'écriture. » Cet «enseignement primaire rénové» nécessite des enseignants mieux payés et mieux formés. Paul Rollin constate pour sa part «[...] des horaires de plus en plus lourds et un volume à acquérir en perpétuelle croissance». Avant même le séminaire, Jospin est donc sensibilisé à la question des contenus, quatrième sujet abordé ce jour-là. Lorsque le ministre demande, au terme d'échanges où s'affiche un consensus, «On a vraiment besoin de revoir les horaires et les programmes? ${ }^{48}$, Jean Geoffroy, conseiller introducteur du sujet, lui répond : «La réponse est clairement oui. Tout le monde, y compris les syndicats, en a conscience. Pas pareil selon les matières. Importance des passerelles. C'est aussi la réflexion sur le contenu du secondaire par rapport à ce qui sera nécessaire dans le supérieur».

Quant à savoir qui pourrait déterminer le contenu des programmes, Allègre défend I'idée que « seuls ceux qui ont la plus grande connaissance des matières ont la capacité de dire ce qu'il faut dans les programmes, à condition qu'ils soient conscients du niveau des étudiants. On va engager la discussion sur les mathématiques et l'économie». Un autre conseiller s'interroge sur «Comment redéfinir les humanités au XXIe siècle? Pour ne pas soumettre l'Éducation à l'économie [...] Pourquoi ne pas confier cette tâche, sous l'autorité du ministre, à de très grandes personnalités (obligées de se confronter)». Olivier Schrameck ajoute alors «si on crée une mission, croiser les compétences avec les gens de terrain (profs de lycée par exemple)». Ce séminaire du 24 juin 1988 voit donc s'ébaucher le futur conseil des programmes, tandis que Claude Allègre affirme, selon les notes prises par le ministre : «les horaires = les alléger, parascolaire à côté ; les programmes = les alléger ; les maîtres = les former, les payer; les techniques modernes = les utiliser ».

Après la rentrée 1988, un second séminaire porte sur un document beaucoup plus conséquent (60 pages dactylographiées) rédigé par le conseiller spécial, Faire du neuf avec du vieux. Un programme pour adapter notre éducation aux temps futurs ${ }^{49}$. La question des contenus d'enseignement, "source principale de dysfonctionnements de notre système éducatif» (page 33), y figure en bonne place car «les programmes scolaires (surtout au niveau du secondaire) sont encombrés et surchargés. L'enseignement extensif a pris le pas sur toute autre

48 Jospin avait écrit en marge de la note de Paul Rollin : «Sommes nous prêts à réellement agir sur ce sujet? Comment? Dans quelle direction?".

49 AN 20040313/2 
forme». Or, il s'agit de «penser le problème globalement et non par cycle. Ceci se traduit notamment par la mise en place de comité de programmes verticaux allant de l'école maternelle à l'université.» (p. 33). Allègre précise que "Les programmes resteraient nationaux : il s'agirait surtout de programmes d'objectifs et de programmescadres dans lesquels pourraient se développer l'innovation pédagogique. Leur conception relèvera d'une commission dont la composition sera modifiée périodiquement statutairement. Elle ne serait plus confiée aux inspecteurs généraux» (p. 31). II ajoute un peu plus loin que «ces programmes allégés seraient définis d'abord par leurs objectifs. Les contenus seraient définis comme des cadres dans lesquels les initiatives pédagogiques pourraient se développer. Les horaires d'enseignement magistral en classe seraient allégés. [...] La vérité, c'est que nous voulons des enfants qui sont [sic] mieux formés et qui aient de meilleures connaissances. En focalisant l'enseignement sur quelques thèmes, en privilégiant l'apprentissage de mécanismes de pensée, en dégageant du temps pour le travail personnel, notre objectifunique est l'élévation du niveau de chacun et de tous» (p. 37). Pour lui, ces comités de programmes verticaux, animés par "une personnalité de haut niveau, dominant sa discipline et ayant montré des capacités et de l'intérêt pour les problèmes d'enseignement», doivent être composés d'autres personnalités de spécialités différentes, mais aussi d'« enseignants actifs de tous les niveaux [...] connus pour leur excellence pédagogique», «un ou deux inspecteurs généraux pour assurer la continuité» : «Exercice de praticiens, la confection des programmes devra rester aux mains des praticiens» (p.39). Néanmoins, Allègre prévoit des instructions à destination de l'ensemble des commissions (disciplines et cycles d'enseignement) : "Les objectifs devront être définis d'abord comme des contributions à la formation de la pensée. L'acquisition de méthodes de travail et de pensée devra être privilégiée sur l'acquisition des connaissances» (Ibid.). Il leur faudra "alléger les programmes, les cours magistraux et favoriser dès les plus jeunes âges I'intérêt, l'initiative, la recherche personnelle. [...] définir les modes d'enseignement souhaitables (travaux pratiques, cours magistraux, horaires groupés ou non) et les modalités de contrôle et d'examen » (lbid.).

Cet argumentaire exposé dans un texte qui n'a pas vocation à devenir public crédite son auteur d'une certaine sincérité. Ce qui ne l'empêche pas de voir l'intérêt budgétaire de la diminution des horaires et des programmes : «Bien que la motivation première de la réduc- il est clair qu'elle va avoir une conséquence importante sur les nombres de postes à pourvoir». (p. 41). Mais, «Naturellement cette opération est difficile à réussir. Car nous risquons d'être accusés d'abaisser le niveau ou de ne pas garder les enfants suffisamment au collège et au lycée. II importe doncque d'une part notre garantie scientifique soit imparable» et que "les commissions de réforme des programmes [...] doivent toutes être portées par des personnalités incontestées sur le plan scientifique et littéraire» (p. 55). Il introduit alors l'exemple des deux commissions tout juste créées, celles de mathématiques et d'économie, dont la présidence est confiée respectivement à Didier Dacunha-Castelle et Edmond Malinvaud.

Lors de ce second séminaire, un consensus apparaît sur le nécessaire allègement des programmes, «leur lourdeur et leur non-interdisciplinarité est un nonsens scientifique» dit même Daniel Bancel. Cependant, chacun mesure qu'il s'agit de faire preuve de prudence et d'éviter de parler publiquement d'allègements, du moins de prime abord. Prudente est d'ailleurs l'attitude générale du ministre qui découvre les problématiques scolaires en ce début d'été 1988. Le rôle de son conseiller spécial Claude Allègre paraît central pour tout ce qui a trait à la réflexion pédagogique, bien qu'il ne soit pourtant pas un expert. Sylvie Aebischer parle même à son propos d'«appétence pédagogique plutôt que d'une expertise consolidée en la matière», de «savoir pratique, intuitif, peu réflexif » (2012, p.73-74).

Cette position sur les contenus d'enseignement, leur importance et leur nécessaire transformation, n'est pas spécifique aux membres du cabinet Jospin. De son côté, le 28 juin 1988, l'Académie des sciences a adressé au nouveau ministre l'introduction au rapport de la «Commission exploratoire sur le système éducatif », créée en interne par autosaisine. "À notre époque de mutations rapides, nous avons besoin davantage de têtes bien faites que de têtes bien pleines; nous devons donc nous attacher à la formation de l'esprit, et pour cela baser notre enseignement sur le travail personnel de l'élève, travail en classe à partir d'un programme léger mais approfondi, et culture acquise par la lecture d'une documentation personnelle $»^{50}$.

Aux deux commissions initialement créées pendant l'été se sont ajoutées en septembre celles de biologie, chimie, physique, sciences de la terre et de l'univers, français, histoire-géographie, enseignements artistiques. Mais pour Allègre, il manque encore un élément

50 Lettre de M. Choquet, professeur émérite à Paris Sud, membre de l'Académie des sciences. AN 20040313/2 
dans l'architecture d'ensemble, une commission transversale, dite «horizontale», chargée d'assurer la cohérence d'ensemble du dispositif et de consolider la légitimité de la démarche. Elle va voir le jour sous la responsabilité de deux personnalités du Collège de France, Pierre Bourdieu et François Gros, à qui le ministre fait appel dans un courrier du 2 novembre $1988^{51}$. Cette fois, ce n'est pas l'institution en tant que telle qui est sollicitée mais certains de ses membres à titre personnel.

À la différence de l'expérience de 1985, le travail des commissions est très balisé par Allègre, et piloté par Luc Soubré et lui-même : "Il serait souhaitable que vous indiquiez quelles réductions de programme et d'horaires vous préconisez pour la rentrée 1989. Il est clair qu'il s'agira d'un effort d'ensemble, coordonné, qui portera sur relativement peu d'heures mais qui aura une valeur symbolique. L'objectif terminal sera de mettre en œuvre une réforme complète des programmes et horaires pour la rentrée $1990 » 52$.

Dès le 26 novembre, une table ronde réunit Allègre, Bourdieu et des membres de commissions pour réfléchir au « redécoupage éventuel des disciplines des Sciences humaines $»^{53}$. De grandes figures intellectuelles, scientifiques internationalement reconnus pour certains, Bouveresse et Derrida, Lévi-Strauss, André Miquel, Maurice Agulhon sont invités. II en ressort une plus grande intégration disciplinaire : les sciences sociales rejoignent la commission histoire-géographie, la commission français devient «langues et littératures » présidée par JeanClaude Chevalier, ancien président de la commission français sous Savary, et Bernard Lortholary et non plus par Bertrand Poirot-Delpech, une commission «philosophie-épistémologie » est créée, co-présidée par Derrida et Bouveresse.

Enfin, pour médiatiser le processus, Jospin présente à la presse le dispositif de rénovation des contenus d'enseignement le 12 décembre 1988.

\section{La naissance du CNP : un dispositif de légitimation à l'efficacité relative}

On le voit, les scientifiques membres des commissions, s'ils fournissent toujours une caution et une légitimité au processus engagé, disposent comparativement à

51 Lettre de L. Jospin à P. Bourdieu, 2 novembre 1988. Archives personnelles de P. Bourdieu

52 Lettre de C. Allègre à P. Bourdieu, 18 novembre 1988. Archives personnelles de P. Bourdieu

53 Lettre de C. Allègre à P. Bourdieu, Ibid.
1985 de beaucoup moins d'autonomie. Alors même qu'ils développent des arguments pédagogiques, la diminution envisagée des horaires et programmes, entre fuites et rumeurs, disqualifie d'emblée leurs travaux, malgré des tentatives de contre-feux ${ }^{54}$. Dans cette faille, vont s'engouffrer les opposants de tous bords, au premier rang desquels le SNES, dont le congrès a lieu à Dijon en mars 1989, qui s'emploie efficacement à mobiliser ses troupes en janvier et février.

Lors de la première table ronde sur le projet de loi d'orientation, le 17 janvier 1989, Lionel Jospin présente le domaine d'actions «des contenus d'enseignement rénovés et des parcours de la réussite», largement inspiré des temps de réflexion collectifs antérieurs du cabinet. Il déclare alors : "L'évolution des connaissances et des métiers rend nécessaire de moderniser les contenus d'enseignement. L'examen en a été engagé grâce à des missions thématiques confiées à d'éminents professeurs et scientifiques. La mise à jour des contenus d'enseignement sera conçue de manière à faire une place importante aux relations entre les disciplines et à permettre le travail en petits groupes ainsi que les recherches personnelles. L'objectif sera de promouvoir un enseignement où l'élève interrogera, réfléchira par lui-même, sera guidé dans son apprentissage.

Les contenus et les programmes seront révisés à intervalles réguliers par un conseil national des programmes d'enseignement. Les programmes qui permettront d'atteindre des objectifs précis au terme de chaque cycle constitueront des cadres nationaux de référence, supports de l'innovation pédagogique et de l'intervention différenciée des enseignants auprès des élèves ${ }^{55}$.

Si le sujet ne mobilise pas ce jour-là les présents à la réunion, le ministre et ses proches doivent pourtant faire face à ce qu'ils nomment une "campagne de désinformation $»^{56}$ et lors de la seconde table ronde le 28 février, Lionel Jospin aborde en premier «les savoirs à transmettre $»^{57}$ dans un exposé complet dont l'essentiel est retranscrit ici dans l'annexe 1, manière de s'opposer

54 Dans une note au ministre datée du 10 février 1989, Soubré suggère une communication type pour «l'expression publique des présidents de commissions». AN 20040313/2

55 Texte introductif à la table ronde du 17 janvier 1989 sur la loi d'orientation. AN 20040313/2

56 Une pochette "Campagne de désinformation - 1989» comprend plusieurs notes dont l'une de L. Soubré à destination de L. Jospin s'intitule «Réponse à la campagne de désinformation du SNES». AN 20040313/2

57 Un document de 24 pages sert d'introduction à la réflexion et aux interventions. Les contenus d'enseignement figurent sur les pages 2-3-4. 
vigoureusement aux rumeurs qui circulent depuis quelques semaines. Pour rassurer les opposants et déminer le terrain, il annonce le report de toute réduction des programmes et des horaires à la rentrée 1990 mais confirme la création du CNP. Il réaffirme aussi l'indépendance des commissions et la possibilité qu'auront tous les partenaires de s'exprimer lors de colloques académiques.

Le dîner de la veille avec les présidents des commissions thématique ${ }^{58}$ a renforcé la détermination du ministre et clarifié la stratégie choisie ${ }^{59}$ : «il y a une démarche libre et réaliste (savants); ily a une consultation du terrain (colloques par académie) ». C'est à cette occasion en effet que Pierre Bourdieu, en accord avec François Gros absent ce soir-là, tous deux très embarrassés par le dénigrement du travail des commissions ${ }^{60}, a$ suggéré au ministre que puissent se tenir des colloques académiques. Ces colloques régionaux seront finalement repoussés à l'automne 1989, après le vote de la loi d'orientation du 10 juillet 1989. Luc Soubré gèrera le dossier depuis le cabinet.

En dépit des difficultés, la commission Bourdieu-Gros ${ }^{61}$ rend son rapport le 8 mars 1989, «Principes pour une réflexion sur les contenus de l'enseignement ». Sept propositions accompagnent le préambule. Le dernier paragraphe traite du CNP : «Le Conseil national des programmes d'enseignement aura pour tâche de mettre en œuvre l'ensemble des principes énoncés cidessus. Ses membres devront être choisis en fonction de leur seule compétence et agir à titre personnel et non en tant que représentants de corps, d'institutions ou d'associations. Il devra travailler en permanence (ce qui suppose que ses membres soient libérés d'une partie de leurs autres charges) pendant une durée de cinq ans, mais les modifications qu'il entendra éventuellement apporter aux programmes en vigueur ne pourront être mises en application que tous les cinq ans. Sa compétence devra

58 Dix-huit personnes y participent, dont quatre conseillers (Schrameck, Goy, Soubré, Allègre) et le directeur des collèges et lycées, André Legrand. AN 2004313/2

59 Notes prises lors du dîner ou à son issue. AN 2004313/2

60 La synthèse des différentes positions des organisations présentes aux tables rondes, recueillies lors de rencontres bilatérales tenues au cours du mois de février, fait état d'une opposition résolue du SNES, de la société des agrégés, du SNALC et du SNLC-FO sur la question des contenus d'enseignement.

61 Onze personnes la composent. Outre les deux présidents, il s'agit de Pierre Baqué, Pierre Bergé, René Blanchet, Jacques Bouveresse, Jean-Claude Chevalier, Hubert Condamines, Didier Dacunha Castelle, Jacques Derrida, Philippe Joutard, Edmond Malinvaud, s'étendre à tous les ordres et à tous les types d'enseignement. ${ }^{62}$

Les commissions verticales remettent, elles, leurs rapports en juin et juillet 1989. Pourtant, ils ne seront rendus publics que quelques semaines seulement avant les colloques, et de manière plutôt confidentielle $^{63}$. La non-diffusion immédiate de ces travaux, l'absence même d'accusé de réception, sans justification, va grandement contrarier leurs auteurs qui vont réagir pour certains très explicitement. Ainsi, JeanClaude Chevalier présente sa démission dans un courrier à Lionel Jospin et explique pourquoi : "C'est la première fois que je suis ainsi rebuté et j'en ressens l'affront pour tous mes collègues qui se sont dépensés sans compter-et sans jamais en recevoir le moindre avantage. Mais je suis surtout extrêmement affecté que nos propositions, éventuellement amendées, ne soient pas soumises aux collègues qui vont se réunir pendant les mois de novembre et de décembre pour des débats pédagogiques. [...] les réactions de nos collègues à nos suggestions auraient été capitales pour l'élaboration d'une politique des contenus destinée à trouver un large accord. Il est vrai que pour ces rencontres, pour le questionnaire qui a été lancé, nous n'avons été, à aucun moment consultés. " ${ }^{64}$

Ce manque de considération leur est d'autant plus insupportable que le ministre semble privilégier les syndicats qu'il reçoit sans cesse. Le sentiment d'avoir été instrumentalisé par le pouvoir politique domine, alors même que les critiques virulentes de certains syndicats, SNES, SNALC et SNLC-FO alliés en la circonstance, dénient toute légitimité à leurs travaux.

Pris en tenailles entre ce qui est perçu comme une campagne de désinformation et une forme de mépris ministériel, Pierre Bourdieu n'est pas davantage satisfait par le travail des commissions thématiques resté trop disciplinaire. La synthèse qu'il souhaitait publier, avec un redécoupage des champs disciplinaires, en "cinq grandes unités», lui apparaît impossible à réali-

62 «Principes pour une réflexion sur les contenus d'enseignement ", Commission présidée par Pierre Bourdieu et François Gros mars 1989, p. 14.

63 Une note émanant de la Direction de l'information et de la communication (DIC), est envoyée à l'automne aux recteurs. Il est précisé que ces «rapports des missions de réflexion sur les enseignements [...] sont des contributions qui n'engagent que leurs auteurs». Un exemplaire de chacun des rapports sera acheminé dans les centres régionaux et départementaux de documentation pédagogique "dans les prochaines semaines» où ils seront consultables.

64 Lettre de J.-C. Chevalier à L. Jospin, 22 septembre 1989. Archives personnelles de P. Bourdieu 
ser. À la fin mai 1989 pourtant, il a adressé aux présidents des commissions et à Claude Allègre un courrier très explicite en ce sens. Après avoir réaffirmé «qu'un des principes majeurs du renouvellement des contenus et des méthodes d'enseignement consiste dans la mise en question des disciplines (de leur existence en certains cas, de leurs frontières en d'autres cas) », il prévient en effet ses collègues qu'il ne pourra que se «désolidariser par avance d'un rapport final qui accepterait en fait ces divisions ${ }^{65}$. Rappelant ce qui aurait dû être le seul fondement de la légitimité d'un tel dispositif de réflexion sur le contenu, il ajoute alors ce qui s'apparente à un constat d'échec : «comment, en effet, combattre le corporatisme professoral qui est, on le sait bien, un des obstacles majeurs au renouveau pédagogique, si une commission qui prétend ne reconnaître d'autres principes que ceux de la science cède par avance sur un point de cette importance? "66. Face à ces déceptions successives, Pierre Bourdieu se désengage progressivement et là, il n'assure pas la promotion du rapport comme en 1985, il n'intervient ni dans la presse écrite ni à la télévision alors même que le rapport porte cette fois son nom et non celui de l'institution Collège de France.

La loi d'orientation est présentée et débattue au Parlement au printemps $1989^{67}$, après une dernière table ronde le 30 mars 1989, peu constructive, les positions des uns et des autres se durcissant et paraissant trop hétérogènes pour être conciliées ${ }^{68}$. Alors que le rapport Bourdieu-Gros vient d'être diffusé, le ministre affirme alors : «La commission ne m'engage pas. Mais voyez la démarche ${ }^{69}$. Depuis janvier, des grèves se sont succédé, mêlant des revendications liées à la fois au dossier de revalorisation et au projet de loi d'orientation, dans lesquelles la question des contenus d'enseignement est utilisée comme exemple de la volonté gouvernementale de casser l'école et d'abaisser le niveau. Pour répondre à ces tensions et à l'inquiétude de l'opinion, Jospin et Rocard participent ensemble à

65 Lettre du 29 mai 1989 aux présidents des commissions thématiques ainsi qu'à F. Gros et C. Allègre. Archives personnelles de P. Bourdieu.

66 Ibid.

67 Le texte définitif est adopté le 4 juillet, l'article 5 du projet devenant l'article 6. Un amendement accepté prévoit que «les avis et les propositions du Conseil seraient rendus publics afin d'assurer une indispensable transparence aux travaux de cette commission ». AN 20020356/1

68 Jospin note en introduction : «Diversité des préoccupations, la force de vos contradictions [...] je dois tenir compte de la diversité des points de vue exprimés». AN 20040313/2

69 Table ronde du 30 mars 1989. AN 20040313/2 une émission télévisée le 8 mars 1989. Ce qui est insuffisant pour calmer le jeu.

Le CNP verra néanmoins le jour. Le cabinet y travaille et dès le printemps 1989, des principes sont fixés, si I'on se fie à la note de Paul Rollin préparée pour l'audition du ministre le 23 mai à l'Assemblée nationale : «Un décret d'application est en préparation. [...] Les rôles de chacun (conseil national, directions pédagogiques du ministère et inspections générales) sont clairement définis. Aux premiers, les réflexions d'ensemble, la définition des grandes orientations, le suivi de l'évolution des disciplines et de leur association en des champs disciplinaires ainsi que les conséquences à tirer de l'émergence de nouvelles disciplines; aux secondes, l'élaboration des programmes avec le concours de groupes de travail associant toutes les compétences; à l'inspection générale, l'évaluation de leur mise en œuvre, de "leur exigibilité et de leur transmissibilité", pour reprendre les expressions employées par MM. Bourdieu et Gros »70.

Dès l'été, le cabinet engage l'ultime étape de consultation par un courrier aux recteurs daté du 31 juillet $1989^{71}$. Les colloques régionaux se tiendront pendant le $1^{\text {er }}$ trimestre 1989-1990, avec des contraintes («procéder à une large consultation», «ménager un temps d'exploitation de celle-ci», «en approfondir les conclusions dans le cadre de colloques régionaux»), mais avec une seule obligation, s'appuyer sur le questionnaire fourni par le ministère. Chaque académie peut donc s'organiser comme elle l'entend dans le cadre posé. Une note méthodologique ${ }^{72}$ est cependant fournie «comportant les orientations qui ont été retenues, un certain nombre de propositions d'organisation faites à titre indicatif et des précisions concernant les moyens qui seront mis à votre disposition ». Le projet de questionnaire est adressé à titre confidentiel. Le

\section{AN 20040313/2}

71 Tous les éléments qui suivent ont été trouvés dans les archives du rectorat d'Orléans-Tours, mis à notre disposition par Florian Marie, l'archiviste, que nous remercions.

72 Le calendrier précise que les colloques devront être organisés un vendredi et un samedi, entre les 24-25 novembre et les 8-9 décembre; la consultation locale devra associer les enseignants, les élèves, les parents, les élus, les partenaires socio-économiques, les associations...et durera au minimum 4 semaines. L'information des médias est laissée au local. Le national se charge du questionnaire et de son envoi, accompagné du rapport Bourdieu-Gros, de la grille d'exploitation des réponses qui servira à l'équipe académique chargée du dépouillement.

Des crédits «communication » sont prévus, de 30000 à 50000 francs par académie, ainsi que des HSA, de 5 à 15 par académie, «pour la constitution d'équipes de consultants et pour les équipes chargées d'exploiter les questionnaires». 
ministre doit être informé du choix des présidents et rapporteur du colloque.

Le calendrier est présenté lors de la réunion des recteurs le 14 septembre $1989^{73}$. II sera officialisé le 21 septembre, soit deux jours après le lancement de la consultation nationale sur les contenus d'enseignement, lors d'une conférence de presse au ministère. 800000 exemplaires d'un questionnaire de 16 pages sont envoyés, base de la consultation dans les établissements avant les synthèses préparatoires aux vingt-huit colloques. Dans le même temps, un courrier identique du ministre est envoyé aux parlementaires, aux présidents de conseils généraux et régionaux, à ceux des conseils économiques et sociaux régionaux, aux inspecteurs d'académie, aux présidents d'université, aux maires, aux inspecteurs de l'information et de l'orientation... On peut supposer que Luc Soubré en charge du dossier au cabinet a largement contribué à l'élaboration du questionnaire : une note du 4 juillet 1989, «Projets de questions pour les colloques régionaux», accompagne ainsi le même jour celle qu'il destine au ministre sur l'organisation de ces colloques ${ }^{74}$. De fait, ni Pierre Bourdieu ni François Gros, ni aucun des présidents des commissions n'ont été apparemment associés à sa réalisation.

Les colloques ont finalement lieu entre les 24/25 novembre et les 15/16 décembre. Les synthèses exigées des présidents et rapporteurs des colloques académiques sont présentées au ministère lors d'une ultime réunion les regroupant tous le 25 janvier 1990, chacun constatant que pendant ces consultations les questions abordées ont largement débordé le cadre du questionnaire, laissant finalement de côté l'évocation des contenus d'enseignement. II devient dès lors impossible d'élaborer une synthèse nationale conséquente à destination du futur CNP, et seul un document de cinq pages et demie sera diffusé dans les académies par la Direction de l'information et de la communication.

73 Deux vagues de diffusion sont prévues, fin septembre et tout début octobre. La grille de dépouillement des questionnaires (24 p.) a été établie par la DEP; elle est envoyée le 27 septembre, avec un mode d'emploi de 6 pages.

Pour répondre aux critiques sur le calendrier jugé par tous beaucoup trop serré, Luc Soubré reporte l'envoi des synthèses régionales au 10 janvier 1990, le retour des questionnaires ayant été repoussé au 20 novembre; il formule à cette occasion des recommandations : synthèse régionale de maximum 20 à 25 pages; indiquer les pourcentages de réponses individuelles et collectives, celles des conseils d'administration; fournir un dossier complet sur la préparation et le déroulement du colloque; archiver au rectorat tous les questionnaires retournés; rappel que les élèves et les associations de parents doivent être représentés.
S'agit-il là d'un échec symbolique et politique du dispositif qui devait asseoir la légitimité du CNP? L'ensemble du processus engagé depuis l'été 1988 semblait voué à ne satisfaire personne, tant l'imbrication des sujets, transformations statutaires, revalorisations, loi d'orientation avec les transformations attendues, obérait tout consensus, dans un milieu professionnel marqué par des cultures professionnelles disparates. Le rôle dévolu aux scientifiques dans ce dernier épisode, pris malgré eux dans des contradictions et conflits sur lesquels ils n'ont aucune prise, leur laisse un goût amer.

Lionel Jospin installe le CNP le 6 mars 1990 après la parution du décret le 23 février. La composition du CNP est arrêtée officiellement le 27 février 1990 : il comprend vingt-deux membres, dont dix-sept appartiennent à l'Éducation nationale, dix étant dans le supérieur et la recherche. Le premier degré ne compte qu'un membre. Les membres sont nommés à titre personnel et ne représentent qu'eux-mêmes. Nommé par arrêté, le premier président est Didier Dacunha-Castelle, président de la commission mathématique, et proche de Claude Allègre. Comme lui, plusieurs membres sont issus des commissions Bourdieu-Gros (Pierre Baqué, Edmond Malinvaud) et ont également participé à l'organisation d'un colloque académique (cas de Bernadette Bornancin) ${ }^{75}$.

Malgré un cheminement discontinu, l'idée d'une commission de révision périodique des contenus d'enseignement, "jouant un rôle de régulation transversale» (Raulin, 2006, p.63), s'est concrétisée et au final plutôt vite. Les constats convergents émis par les uns et les autres entre 1983 et 1990 laissent à penser que la création de l'instance CNP répondait, malgré les critiques et sa légitimité malmenée, à un besoin partagé de décentrement : le sujet sensible des programmes ne pouvait plus être traité seulement par l'administration ministérielle et les associations disciplinaires, par des acteurs à la fois juges et parties. Le choix politique d'élargir à des universitaires, personnalités scientifiques reconnues unanimement pour leurs compétences, s'est imposé dans les périodes Savary-Mitterrand et Jospin. Cependant, les contradictions inhérentes à un dispositif oscillant entre autonomie et

75 Certaines personnalités ont ainsi participé à tout ou partie des commissions des années 1980, avant d'entrer pour cinq années au CNP. Cette observation mériterait d'être approfondie, y compris par des entretiens avec ces acteurs-là. La désignation des membres du CNP a été débattue entre les principaux membres du cabinet de Jospin, comme la note confidentielle de Paul Rollin du 25 octobre 1989 le met en évidence AN 19930637/8. 
instrumentalisation ont mis en porte à faux les personnalités engagées tandis que dessaisir d'une certaine façon les inspections générales et les associations disciplinaires de leurs prérogatives antérieures, a modifié les rapports de force. La légitimité des savants impliqués n'a pas suffi à contrer les tensions générées par cette rupture dans les pratiques, dans un contexte par ailleurs complexe de désaccords syndicaux vis-à-vis de la loi d'orientation en discussion et de la revalorisation des enseignants. Le travail en commissions verticales, regroupant parfois plusieurs disciplines, et en commissions horizontales, par segments scolaires, ainsi que la rédaction de rapports ont transformé les processus aboutissant à des changements de programme, dans ses modalités comme dans ses temporalités. Désormais la réforme de l'ensemble des disciplines sera globale, coordonnée et simultanée, avec des principes communs définis par le CNP, en lien avec le cabinet du ministre et les directions pédagogiques du ministère.

À partir de 1990, le CNP tentera de jouer le rôle assigné dans la loi d'orientation mais de fortes turbulences contrarieront son fonctionnement. L'arrivée à sa présidence de Luc Ferry fin 1993 permettra de le stabiliser jusqu'en 2002 où il le quitte pour devenir ministre de l'Éducation nationale. La loi Fillon d'avril 2005 dans son article 15 supprime le CNP, qu'elle remplace par le Haut Conseil de l'éducation, lui-même dissous dans la loi de refondation de l'école de la République de Vincent PeilIon en 2013 (article 32). Depuis, c'est le Conseil supérieur des programmes qui est chargé de l'élaboration des programmes. Objet lui-même de controverses, il peine à asseoir sa légitimité, alors même que les contenus d'enseignement ne paraissent pas avoir permis une transformation des pratiques enseignantes.

L'idée d'ouvrir et de réguler autrement le choix des contenus d'enseignement paraît pourtant installée dans le paysage éducatif malgré les aléas des instances qui en ont eu la charge. II reste à en comprendre et analyser la genèse et le fonctionnement, à les comparer, à rechercher une quelconque continuité, tant dans leur composition que dans les objectifs assignés et les productions effectives.

Lydie Heurdier Université Paris 8, Laboratoire CIRCEFT-ESCOL Iheurdier@gmail.com

Pierre Clément Université de Rouen-Normandie, CIRNEF pierclement@gmail.com

\section{Bibliographie}

AEBISCHER S. (2012). «Réinventer l'école, réinventer l'administration. Une loi pédagogique et managériale au prisme de ses producteurs ». Politix, n98, p. 57-83.

ALIX P., D'ENFERT R. \& GISPERT H. (2015). «Des commissions pour réformer les disciplines (1945-1980)». In R. D'Enfert \& J. Lebaume [dir.], Réformer les disciplines. Les savoirs scolaires à l'épreuve de la modernité, 1945-1985. Rennes: Presses universitaires de Rennes, p. 85-111.

BALLAND L. (2009). Une sociologie politique de la crise de l'École. De la réussite d'un mythe aux pratiques enseignantes. Thèse de doctorat, sciences politiques : université Paris X-Nanterre.

CHAPOULIE J.-M. (2006). "Les nouveaux spécialistes des sciences sociales comme "experts" de la politique scolaire en France 1945-1962». Genèses, n 54 , p. 124-145.

CHAPOULIE J.-M. (2010). L'École d'État conquiert la France. Deux siècles de politique scolaire. Rennes: Presses universitaires de Rennes.

CHAPOULIE J.-M. (2015). "Postface». In R. D’Enfert \& J. Lebaume [dir.], Réformer les disciplines. Les savoirs scolaires à l'épreuve de la modernité, 1945-1985. Rennes : Presses universitaires de Rennes, p.237-243.

CLÉMENT P. (2012). «Le Conseil national des programmes (1985-1994) : I'institutionnalisation chaotique d'une entreprise réformatrice». Politix, n 98, p. 85-107.

CLÉMENT P. (2013). Réformer les programmes pour changer l'école? Une sociologie historique du champ du pouvoir scolaire. Thèse de doctorat, sociologie : université de Picardie Jules Verne.

D’ENFERT R. \& KAHN P. (2011). Le temps des réformes. Disciplines scolaires et politiques éducatives sous la Ve République. Les années 1960. Grenoble : Presses Universitaires de Grenoble.

D'ENFERT R. \& LEBAUME J. [dir.] (2015). Réformer les disciplines. Les savoirs scolaires à l'épreuve de la modernité, 19451985. Rennes: Presses universitaires de Rennes

DONEGANI J.-M. \& SADOUN M. (1976), «La réforme de l'enseignement secondaire en France depuis 1945 : analyse d'une non-décision ». Revue française de science politique, vol.26, $n^{\circ} 6$, p. $1125-1146$.

DREYFUS F.-G. (1965). «Un groupe de pression en action : les syndicats universitaires devant le projet Billères de réforme de l'enseignement (1955-1959). Revue française de science politique, vol. 15, n², p. 213-250.

DUBOIS V. (1999). La politique culturelle. Genèse d'une catégorie d'intervention publique. Paris : Belin.

DUBOIS V. (2003). «La sociologie de l'action publique. De la socio histoire à l'observation des pratiques ». In P. Laborier \& D. Trom [dir.], Historicités de l'action publique. Paris : PUF, p.347-364.

GAUTHIER R.-F. (1988). Querelles d'école, pour une politique des contenus d'enseignement. Paris : SERDIMAP et Hatier.

GAUTHIER R.-F. (2014). Ce que l'école devrait enseigner. Pour 
une révolution de la politique scolaire en France. Paris : Dunod.

GEAY B. (2005). Le syndicalisme enseignant. Paris : La Découverte.

GUSFIELD J. (2009). La culture des problèmes publics. L'alcool au volant : la production d'un ordre symbolique. Paris : Economica.

LASCOUMES P. \& LE GALÈS P. [dir.]. (2004). Gouverner par les instruments. Paris : Presses de Sciences-Po.

LELIEVRE C. \& NIQUE C. (1995). L'École des présidents. De Charles de Gaulle à François Mitterrand. Paris : Odile Jacob.

MAYEUR J.-M. (1985). «La guerre scolaire, ancienne ou nouvelle histoire?». Vingtième siècle, ${ }^{\circ}{ }^{5}$, p. 101-110.

MEURICE J.-M. (2011). "La véritable histoire des origines d'ARTE par l'un de ses créateurs ». Télévision, n², p. 35-51.

PAIR C. (1986). Rue du bac. Une nouvelle donne pour l'école. Paris: Syros.

PROST A. (1983). «Les lycées et leurs études au seuil du XXI siècle». Rapport du groupe de travail national sur les seconds cycles présidé par Antoine Prost. Paris : ministère de l'Éducation nationale.

RAULIN D. (1996). «L'élaboration des programmes du secondaire». Revue internationale d'éducation de Sèvres, $\mathrm{n}^{\circ} 12$, p. $99-103$
RAULIN D. (2006). «De nouveaux rapports entre science et politique : le cas des programmes scolaires». Revue française de pédagogie, $\mathrm{n}^{\circ} 154$, p.61-71.

ROBERT A. (2004). «Jeux croisés des syndicats d'enseignants face aux réformes et projets de réforme (1944-2000)». In J. Girault [dir.], Les enseignants dans la société française au XXe siècle. Paris : Publications de la Sorbonne, p. 113-132.

ROBERT A. (2015). L'école en France de 1945 à nos jours. Grenoble : Presses universitaires de Grenoble.

SCHWARTZ L. (1981). La France en mai 1981 : l'enseignement et le développement scientifique. Paris : La Documentation française.

SCHWARTZ L. (1997). Un mathématicien aux prises avec le siècle. Paris : Odile Jacob.

TISSOT S. (2007). L'État et les quartiers. Genèse d'une catégorie de l'action publique. Paris : Éd. du Seuil.

TOPALOV C. [dir.]. (1999). Laboratoires du nouveau siècle. La nébuleuse réformatrice et ses réseaux en France (1880-1914). Paris : Éditions de l'École des hautes études en sciences sociales. 
Extraits de l'intervention de Lionel Jospin en ouverture de la table ronde du 28 février 1989

(Source : AN 20040313/2)

“Nous en sommes à la phase première, celle de la réflexion. Réflexion libre, je l'ai voulue. Elle a été confiée à Pierre Bourdieu et à François Gros, à des spécialistes incontestés de leurs disciplines et à des praticiens de l'Éducation, professeurs et instituteurs notamment. [...] Ces réflexions ont donné lieu à des rumeurs variées et diverses dont la multiplicité et la fantaisie ont de quoi inquiéter les esprits qui attribuent à notre milieu éducatif des vertus de rigueur intellectuelle, de vérification des sources, d'ouverture d'esprit et de sens des évolutions. [...]

$1^{\circ}$ Pierre Bourdieu, François Gros et leurs collègues sont en train d'élaborer un texte définissant les principes qui, estiment-ils, devraient inspirer les manières de réformer les contenus des enseignements et donc guider le travail des commissions thématiques. Cette charte va être publiée et chacun pourra se rendre compte de l'esprit dans lequel ces commissions ont travaillé.

Parmi ces recommandations, il en est une que je propose d'inscrire dans la loi qui est la création d'un comité consultatif des programmes et des contenus. Ce comité composé de personnalités choisies uniquement en fonction de leurs compétences sera l'instance qui conseillera le ministre pour l'élaboration des programmes. Ceci avec un double souci : éviter les révisions trop fréquentes, ménager avant les révisions éventuelles un temps de préparation suffisant.

$2^{\circ}$ Le groupe Bourdieu-Gros va entamer maintenant une 2e phase, qui sera toujours une phase de réflexions, beaucoup plus tournée vers la question des contenus intrinsèques. [...] Parallèlement à cette véritable recherche, nous allons organiser sur la suggestion de Pierre Bourdieu et François Gros, une série de colloques régionaux, qui discuteront des contenus des enseignements. Y participeront tous les partenaires du système éducatif, professeurs, instituteurs, étudiants, élèves, parents d'élèves, milieux socioprofessionnels. Nous tirerons les leçons de ces colloques au cours d'une réunion de synthèse à Paris. Ainsi chacun sera informé et associé. La transparence sera assurée.

À partir de là, mais à partir de là seulement, un processus progressif de décision pourra être mis en œuvre. Il le sera naturellement en respectant les concertations habituelles. Ce processus prendra naturellement du temps et en particulier aucun changement ne pourra intervenir pour la rentrée 1989. [...] Je le confirme ici : I'EPS et les disciplines artistiques relèvent toutes de l'enseignement obligatoire. [...] J'ai demandé à une personnalité, M. Alain Hébrard, de faire connaître à M. Bourdieu les résultats de ses travaux. Il en va de même pour les enseignements artistiques et j'ai demandé à M. Pierre Baqué de faire part à M. Pierre Bourdieu des propositions qu'il a déjà émises à mon intention." 
